\title{
Made for each other
}

This special issue represents the perfect marriage of two of the strongest trends in thoracic surgery today.

On the one hand is the phenomenon of uniportal VATS. It is no exaggeration to say that this approach to chest surgery has taken the world by storm over the past several years, garnering a fervent following across the globe through its promise of being the least invasive approach yet to surgery in the chest. The rapid rise of uniportal VATS would not have been possible without modern media support of operative videos. Most thoracic surgeons first encounter this technique through videos on the internet. They continue to review those videos as they advance their skills with single-port surgery.

This leads us to the second important trend: the establishment of video-based, peer-reviewed surgical journals—of which the Fournal of Visualized Surgery is a leading example. Conventional medical journals tend to shun surgical technique papers as these are often viewed, but rarely cited. However, surgeons know very well the usefulness of such articles in teaching and learning vital operative skills. Video-based journals such as the fournal of Visualized Surgery provide an authoritative platform for surgeons to share such videos of essential skills and techniques which would otherwise not be found in "mainstream" journals. The fact that the articles contained are peer-reviewed further lends credence to the videos that would not be available if they were merely posted on social media.

The synergy of uniportal VATS and the Fournal of Visualized Surgery is therefore uniquely appropriate. A video-propagated technique is featured in a video-based journal. What readers will discover within these pages—and the associated videos-is a visual demonstration of the best techniques and latest viewpoints surrounding the uniportal approach. Dr. Migliore-himself a pioneer of the single-port technique-has assembled a formidable team of experts in the field to author the articles. There is no doubt that every reader with an interest in uniportal VATS will find something to intrigue or inspire here.

\section{Acknowledgements}

None.

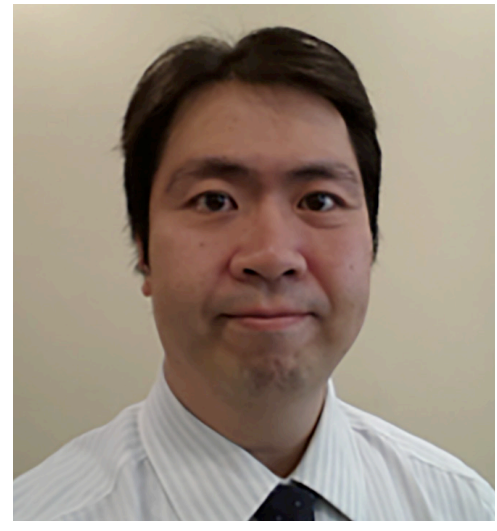

Alan D. L. Sihoe

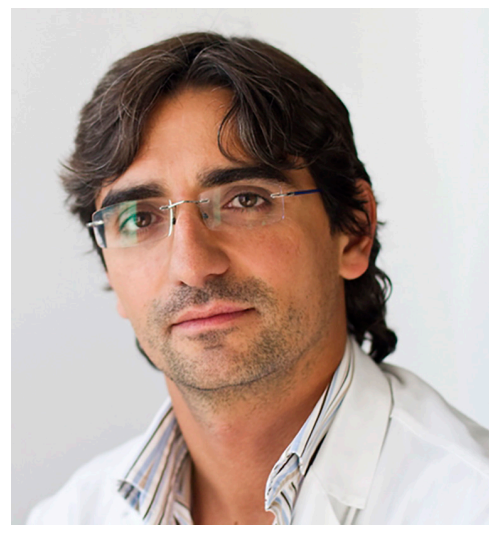

Diego Gonzalez-Rivas 


\section{Alan D. L. Sihoe, MBBChir, MA (Cantab), FRCSEd (CTh), FCSHK, FHKAM (Surgery), FCCP ${ }^{1,2,3}$ \\ ${ }^{1}$ Department of Surgery, The University of Hong Kong, Hong Kong, China; \\ ${ }^{2}$ Department of Surgery, The University of Hong Kong Shenzhen Hospital, Shenzhen 518048, China; \\ ${ }^{3}$ Department of Thoracic Surgery, Tongii University Shanghai Pulmonary Hospital, Shanghai 200433, China. \\ (Email: adls1@hku.hk) \\ Diego Gonzalez-Rivas, MD, FECTS ${ }^{4,5}$ \\ ${ }^{4}$ Director Uniportal VATS training program, Shanghai Pulmonary Hospital, Shanghai 200433, China; \\ ${ }^{5}$ Department of Thoracic Surgery, Coruña University Hospital, Coruña, Spain. \\ (Email: diego.gonzalez.rivas@sergas.es) doi:10.21037/jovs.2018.05.12 \\ Conflicts of Interest: The authors have no conflicts of interest to declare.}

doi: 10.21037 /jovs.2018.05.12

Cite this article as: Sihoe AD, Gonzalez-Rivas D. Made for each other. J Vis Surg 2018;4:96. 